\title{
Acknowledgment to JCMK Editorial Board and Peer-Reviewers for contribution in 2021
}

\section{Laura Dybyssova}

Executive Secretary, on behalf of editorial team of the Journal of Clinical Medicine of Kazakhstan On behalf of the Journal of Clinical Medicine of Kazakhstan, we would like to express our appreciation to all editorial and advisory board members, reviewers and authors who contributed to this journal in year 2021.

\section{EDITORIAL AND ADVISORY BOARD MEMBERS IN 2021}

FOUNDER and HONORED EDITOR

Abay Baigenzhin, (Kazakhstan)

\section{EDITOR-IN-CHIEF}

Abduzhappar Gaipov, (Kazakhstan)

\section{ASSOCIATE EDITORS}

Sinan Kardes, (Turkey)

Ashish Jaiman, (India)

Mathias Hossain Aazami, (Iran)

\section{EDITORIAL BOARD}

Yasin Uzuntarla, (Turkey)

Temirlan Karibekov, (Kazakhstan)

Sakir Ahmed (India)

Saltanat Tuganbekova, (Kazakhstan)

Manarbek Askarov, (Kazakhstan)

Zulfiya Orynbayeva, (USA)

Rimantas Benetis, (Lithuania)

Galina Fedotovskikh, (Kazakhstan)

Ospan Mynbaev, (Russian Federation)

Gunay Akbarova, (Azerbaijan)

Selman Unverdi, (Turkey)

Ulan Kabayev, (Kazakhstan)

Talgat Nurgozhin, (Kazakhstan)

Yuriy Kazakov, (Ukraine)

Almaz Makenjan uulu, (Kyrgyz Republic)

Jakhongir Alidjanov, (Uzbekistan)

Praveen Kumar Potukuchi, (USA)

Oybek Rustamov (Australia)

\section{EXECUTIVE SECRETARY}

Laura Dybyssova (Kazakhstan)

\section{ADVISORY BOARD}

Turgut Teke, (Turkey)

Kubes Jiri, (Czech Republic)

Yaroslav Tolstyak, (Ukraine)

Rustam Mustafin, (Bashkortostan, Russian Federation)

Adem Kucuk, (Turkey)

Yana Sotskaya, (Ukraine)

Ainura Dzhangaziyeva, (Kyrgyz Republic)

Mehtap Tinazli, (Turkey)

Yulia Lunitsyna, (Russian Federation)

Yuksel Ersoy, (Turkey)

Rikhsi Sabirova, (Uzbekistan)

Nurdin Mamanov, (Kyrgyz Republic)

Mariya Derbak, (Ukraine)

Anatoliy Kolos, (Kazakhstan)

Vitaliy Koikov, (Kazakhstan)

Saule Abseitova, (Kazakhstan)

Almagul Kushugulova, (Kazakhstan)

Marlen Doskali, (Kazakhstan)

Kakharman Yesmembetov, (Kazakhstan)

Nelya Bissenova, (Kazakhstan)

Gauri Bapayeva, (Kazakhstan)

Bagdat Imasheva, (Kazakhstan)

Galiya Shaimardanova, (Kazakhstan)

Nasrulla Shanazarov, (Kazakhstan)

Adilzhan Albazarov, (Kazakhstan)

Elmira Chuvakova, (Kazakhstan)

Zhannat Taubaldieva, (Kazakhstan)

Aidos Konkayev, (Kazakhstan)

Samat Saparbayev, (Kazakhstan)

Olga Ulyanova, (Kazakhstan)

Galiya Orazova (Kazakhstan)

Natavan Aliyeva, (Azerbaijan) 
Abay Baigenzhin (Kazakhstan)

Abduzhappar Gaipov (Kazakhstan)

Aathira R (India)

Abraham Adebusoye Tanimowo (Nigeria)

Ahmad Ali (Pakistan)

Ahmed Bilal Genc (Turkey)

Ahmed Cihad Genç (Turkey)

Ahmed Salih Alshewered (Iraq)

Ahmet Taylan Çebi (Turkey)

Aiymkul Ashimkhanova (Kazakhstan)

Akerke Kalimbetova (Kazakhstan)

Alexander Rudenko (Kazakhstan)

Almas Tolegenuly (Kazakhstan)

Alpamys Issanov (Kazakhstan)

Amangali Akanov (Kazakhstan)

Anara Abbay (Kazakhstan)

Andrey Semonenko (Ukraine)

Anton Tkachenko (Ukraine)

Arif Emre (Turkey)

Aritra Saha (India)

Ashish Jaiman (India)

Ashish Joshee (India)

Assel Zhumagulova (Kazakhstan)

Ayşe Karaoğullarından (Turkey)

Ayşe Neslihan Balkaya (Turkey)

Aziz Serkan Senger (Turkey)

Belgin Akan (Turkey)

Burak Sayar (Turkey)

Canbay S. Durmaz (Turkey)

Çetin Murat Altay (Turkey)

Chandeep Singh (India)

Daniya Serdaliyeva (Kazakhstan)

Deniz Şenol (Turkey)

Dina Rybalkina (Kazakhstan)

Dmitriy Viderman (Kazakhstan)

Döndü Üsküdar Cansu (Turkey)

Elzar Matmusaeva (Kyrgyzstan)

Emine Ibici Akça (Turkey)

Emre Bilgin (Turkey)

Emre Bozdag (Turkey)

Emre Yılmaz (Turkey)

Engin Özbay (Turkey)

Ercan Aydin (Turkey)

Erkut Etçioğlu (Turkey)

Ertan Altaylı (Turkey)

Faruk Karandere (Turkey)

Fatih Orhan (Turkey)

Fatih Tanrıverd (Turkey)

Galiya Orazova (Kazakhstan)

Gizem Pehlivan (Turkey)

Gokmen Ozceylan (Turkey)

Gulnar Rakhimbekova (Kazakhstan)

Gulnur Zhakhina (Kazakhstan)

Hilmi Bozkurt (Turkey)

Hristiana Capros (Moldova)

Ibrahim Halil Inanc (Turkey)

İbrahim Kürtül (Turkey)

İlkay Ceylan (Turkey)

Izzuddin Hamzan (Malaysia)

Jamilya Saparbay (Kazakhstan)

Kamilya Kedelbaeva (Kazakhstan)

Karina Nukeshtayeva (Kazakhstan)

Kuat Oshakbayev (Kazakhstan)

Kuralay Atageldiyeva (Kazakhstan)

Leila Razakhani (Iran)

Leonid Sevastyanov (Kazakhstan)
Luigi Gargiulo (Italy)

Mahmut Çay (Turkey)

Maksim Potjagenko (Ukraine)

Malika Khaiitova (Kazakhstan)

Mariya Derbak (Ukraine)

Mariya Dmitriyeva (Kazakhstan)

Matthew Naanlep Tanko (Kazakhstan)

Medet Toleubayev (Kazakhstan)

Metin Yildiz (Turkey)

Mohamad Aljofan (Australia)

Muammer Karakayali (Turkey)

Mujeeb ur Rahman (China)

Mursit Dincer (Turkey)

Mustafa Canbolat (Turkey)

Nasrulla Shanazarov (Kazakhstan)

Natalia Cebotarenco (USA)

Nataliya Chekalina (Ukraine)

Natalya Glushkova (Kazakhstan)

Nilesh Gangan (USA)

Nur Prasetyo Nugroho (Indonesia)

Nurgul Abenova (Kazakhstan)

Olayinka Rasheed Ibrahim (Katsina)

Oleg Gaisenok (Russia)

Owais Ahmed Wani (India)

Özlem Akarsu (Turkey)

Petar Jovan Avramovski (Macedonia)

Prajna Anirban (India)

Raikhan Bolatbekova (Kazakhstan)

Rohat Ak (Turkey)

Rumiyya Zakariyya Karimova (Azerbaijan)

Ruslan Bilal (Kazakhstan)

Sagar Anil Kurkure (India)

Samat Kozhakhmetov (Kazakhstan)

Samet Sayılan (Turkey)

Sana Myrzakhmet (Kazakhstan)

Saniya Saussakova (Kazakhstan)

Sara Çetin Şanlıalp (Turkey)

Saule Balmagambetova (Kazakhstan)

Saule Mussabekova (Kazakhstan)

Seda Beyhan Sagmen (Turkey)

Seda Keskin (Turkey)

Selcuk Kayir (Turkey)

Serdar Abut (Turkey)

Serik Aitaliyev (Kazakhstan)

Sevda Canbay Durmaz (Turkey)

Sezgin Bahadır Tekin (Turkey)

Shiraslan Bakhshaliyev (Turkey)

Shivangi Singh (India)

Sholpan Daniyarova (Kazakhstan)

Sibel Ateşoğlu (Turkey)

Sibel Yaşar (Turkey)

Sinan Kardeş (Turkey)

Srinivasa Bolla (India)

Tayfun Kara (Turkey)

Ubi Essien Isaac (Nigeria)

Ulanbek Balpukov (Kazakhstan)

Ülkü Karaman (Turkey)

Vedat Delibas (Turkey)

Viktoriya Parakhina (Kazakhstan)

Vitaliy Sazonov (Kazakhstan)

Yasemin Kutlu (Turkey)

Yodang Yodang (Indonesia)

Yusuf Alper Sonmez (Turkey)

Zeynep Kizilcik Özkan (Turkey)

Zhenis Sakuov (Kazakhstan)

Zhenisgul Tlegenova (Kazakhstan) 


\section{PEER-REVIEWERS WHO CONTRIBUTED TO THIS JOURNAL IN 2021}

\section{OUR STATISTICS}

Journal of Clinical Medicine of Kazakhstan published 6 regular issues in 2021

- Volume 18, Number 1 (2021) with 17 articles

- Volume 18, Number 2 (2021) with 15 articles

- Volume 18, Number 3 (2021) with 15 articles

- Volume 18, Number 4 (2021) with 17 articles

- Volume 18, Number 5 (2021) with 16 articles

- Volume 18, Number 6 (2021) with 15 articles

During 2021, 84 articles were accepted, 78 articles were rejected, acceptance rate was $52 \%$.

\section{AUTHORS 2021}

Authors and coauthors who contributed to this journal in 2021 were from the following countries: Kazakhstan, Turkey, Ukraine, Russia, Kyrgyzstan, India, USA, Indonesia, Nigeria, Malaysia, Moldova, Iran, Iraq, Australia, Azerbaijan, Italy, Macedonia.

The editorial team of the Journal of Clinical Medicine of Kazakhstan would like to express gratitude for your valuable support and being part of our excellent team. We appreciate your continuous efforts and hope to continue receiving your great feedback, valuable ideas, and interesting scientific papers to further improve the quality and impact of the Journal of Clinical Medicine of Kazakhstan.

Sincerely yours, 